\title{
Dexamethasone-induced hepatomegaly and steatosis in larval zebrafish
}

\author{
Guojun Yin1,2, Liping Cao², Jinliang Du², Rui Jia², Takio Kitazawa', Akira Kubota³ \\ and Hiroki Teraoka 1 \\ ${ }^{1}$ School of Veterinary Medicine, Rakuno Gakuen University, Ebetsu 069-8501, Japan \\ ${ }^{2}$ Key Laboratory of Freshwater Fisheries and Germplasm Resources Utilization, Ministry of Agriculture, Freshwater \\ Fisheries Research Center, Chinese Academy of Fishery Sciences, Wuxi 214081, China \\ ${ }^{3}$ Diagnostic Center for Animal Health and Food Safety, Obihiro University of Agriculture and Veterinary Medicine, \\ Obihiro 080-8555, Japan
}

[Recommended by Chiharu Tohyama]

(Received May 2, 2017; Accepted May 5, 2017)

\begin{abstract}
Fish hepatobiliary syndrome, characterized by hepatomegaly and fatty liver, has been frequently reported in many cultured fish species and has caused a dramatic economic loss in China. Glucocorticoids are thought to be important non-nutritional factors for hepatomegaly and fatty liver development. In the present study, a dexamethasone-induced zebrafish model of fatty liver and hepatomegaly was established, and the role of glucocorticoid receptor (GR) in the development of hepatomegaly and fatty liver was investigated using developing zebrafish. Exposure of larval zebrafish at 5 days post fertilization (dpf) to dexamethasone for $24 \mathrm{hr}$ caused significant increases of liver size and number of fish with hepatic steatosis at $6 \mathrm{dpf}$. The increase of liver size caused by dexamethasone was significantly reversed by treatment with RU486, a GR antagonist, and by gene knock-down with a morpholino against the GR. The dexamethasone-induced hepatic steatosis was also inhibited by treatment with RU486. Overall, the results highlight larval zebrafish as a useful model for stress-induced liver failure.
\end{abstract}

Key words: Glucocorticoid, Hepatomegaly, Liver, Steatosis, Zebrafish

\section{INTRODUCTION}

Glucocorticoids are the most effective anti-inflammatory agents. They are widely prescribed for treatment of inflammation, allergies and autoimmune disorders. Due to increasing needs in medical prescriptions and incomplete removal during wastewater treatment, increasing presence of glucocorticoids in aquatic environments has been reported in China, Europe and USA (Chen et al., 2016). Hence, glucocorticoid contamination has become a major environmental issue.

In mammals, it has been reported that chronically elevated glucocorticoid levels are intrinsically tied to fatty liver development (Vegiopoulos and Herzig, 2007). Studies have shown that glucocorticoids regulate lipid metabolism and induce fatty liver and hepatomegaly (Patel et al., 2011). Studies on glucocorticoids in fish have mainly focused on their effects on carbohydrate metabolism and immune function (Boone et al., 2002; Salas-Leiton et al.,
2012). Effects of glucocorticoids on fatty liver development and hepatomegaly in fish have not been reported.

A recently emerged disease called fish hepatobiliary syndrome, characterized by fatty liver, hepatomegaly and color change, has been frequently reported in many cultured fish species and has caused a dramatic economic loss in China (Liu et al., 2009). It is generally believed that overfeeding of a nutritionally unbalanced diet is one of the main reasons for the development of fatty liver and hepatomegaly. We hypothesized that glucocorticoid contamination may be another reason for the development of fatty liver and hepatomegaly in fish. A stress-induced increase in the blood cortisol level might also be a reason for the development of these liver diseases because fish are sometimes cultured at a high density.

The zebrafish liver can be confirmed by $5 \mathrm{dpf}$ under a conventional microscopy. The transparency of the larvae enables in vivo visual observation of internal organs including the liver under a microscope. Moreover, hepat-

Correspondence: Hiroki Teraoka (E-mail: hteraoka@rakuno.ac.jp) 
ic steatosis upon exposure to some toxins in larvae can be observed by oil red staining (Howarth et al., 2013). The zebrafish has a single gene of the glucocorticoid receptor that is expressed in the liver and intestine by $5 \mathrm{dpf}$ (Bertland et al., 2007). Therefore, the larval zebrafish is an excellent model organism for studying fatty liver and hepatomegaly and the possible involvement of the glucocorticoid receptor in these toxicological endpoints.

Dexamethasone, an artificially synthesized glucocorticoid, has been used to establish models of fatty liver and hepatomegaly in mammals (Thatcher and Caldwell, 1994). It is more potent than cortisol, making it an excellent model analogue for cortisol. Therefore, dexamethasone was used to study the effects of glucocorticoids on liver size and lipid formation.

\section{MATERIALS AND METHODS}

\section{Reagents}

Reagents were of special grade and were purchased from Wako Pure Chemicals (Osaka, Japan) unless otherwise stated.

\section{Zebrafish}

The long-fin wild-type strain of zebrafish was used for all experiments, and the zebrafish were maintained as previously described (Nijoukubo et al., 2016). Adult zebrafish were obtained from a local pet shop. Fertilized eggs were collected from multiple pair of adult zebrafish. Embryos were reared as described previously. We used eggs from the same group of adult fish for all experiments.

\section{Chemical exposure and observation of hepatomegaly}

Zebrafish larvae (5 dpf) were exposed to either dimethyl sulfoxide (DMSO) or different concentrations of dexamethasone dissolved in DMSO in $3 \mathrm{~mL}$ of Zebrafish Ringer solution (38.7 mM NaCl, $1.0 \mathrm{mM} \mathrm{KCl,} 1.7 \mathrm{mM}$ HEPES-NaOH pH 7.2, $\left.2.4 \mathrm{mM} \mathrm{CaCl}{ }_{2}\right)$ in $3.5 \mathrm{~cm}$ diameter polystyrene Petri dishes (Nijoukubo et al., 2016). Twenty-four hours later, zebrafish were placed dorso-laterally in 3\% carboxymethyl cellulose/Zebrafish Ringer solution, and images of $6 \mathrm{dpf}$ larvae were taken under a microscope. Images were imported into Adobe Photoshop 7.0. The region of the liver in the image was manually drawn, and the pixel value of the target region was defined as the liver size.

\section{Gene knock-down with morpholinos}

Splice blocking-type morpholino of the zebrafish glu- cocorticoid receptor (zfGR) (5'-CTGCTTCATGTATTTTAGGGTTCCG-3') (Mathew et al., 2007) was obtained from Gene Tools (Philomath, OR, USA). Standard control morpholino (Std-MO) recommended by Gene Tools was used as a negative control (Nijoukubo et al., 2016). Both morpholinos were diluted in $\mathrm{Ca}^{2+}$-free $\mathrm{ZR}$ solution to the concentrations indicated in Table 1. A Narishige IM-300 microinjector (Tokyo, Japan) with a fine glass needle was used to inject $2 \mathrm{~nL}$ of morpholino into the yolk of 1- to 4-cell stage embryos. These morphants were exposed to dexamethasone at $5 \mathrm{dpf}$ and used for observations at $6 \mathrm{dpf}$.

\section{Oil red staining}

Zebrafish larvae at $7 \mathrm{dpf}$ were fixed overnight in $4 \%$ paraform aldehyde with $\mathrm{PBS}$ at $4^{\circ} \mathrm{C}$. After fixation, samples were washed twice in PBS, followed by oil red $\mathrm{O}$ staining with freshly filtered $0.5 \%$ oil red $\mathrm{O}$ in $60 \%$ 2-propanol for 1-2 hr. Samples were quickly washed a few times in 60\% 2-propanol and then finally washed once in PBS (Raldúa et al., 2008).

\section{Statistical analysis}

All values are shown as means \pm standard error of the mean. Data were analyzed with one-way ANOVA followed by the Tukey-Kramer post hoc test. $P$-values $<0.05$ were considered statistically significant.

\section{RESULTS AND DISCUSSION}

As shown in Fig. 1, exposure of the 5 dpf larval zebrafish to 12.5 and $25.0 \mu \mathrm{g} / \mathrm{mL}$ dexamethasone for $24 \mathrm{hr}$ caused significant increases of liver size. This effect was attenuated by the GR receptor antagonist mifepristone (RU486); co-exposure of the larval zebrafish to RU486 ( 1.5 and $3 \mu \mathrm{g} / \mathrm{mL}$ ) and the dexamethasone reduced dexamethasone-induced increase of liver size, suggesting that

Table 1. Effect of a glucocorticoid receptor antagonist on dexamethasone-induced hepatic steatosis.

\begin{tabular}{lccc}
\hline Treatment & $(-) \%$ & $(+) \%$ & $(++) \%$ \\
\hline Control & 50 & 40 & 10 \\
D12.5 & 0 & 40 & 60 \\
D12.5+ RU 0.75 & 10 & 30 & 60 \\
D12.5+ RU 1.5 & 50 & 50 & 0 \\
D12.5+ RU 3.0 & 60 & 40 & 0 \\
\hline
\end{tabular}

Twenty fish were used in each group. The degree was assessed on the basis of degree of redness in the liver, as indicated in Fig. 3. D12.5: $12.5 \mu \mathrm{g} / \mathrm{mL}$ dexamethasone, RU 0.75, RU 1.5, RU 3.0: RU486 (0.75-3.0 $\mu \mathrm{g} / \mathrm{mL})$. 
Hepatomegaly in larval zebrafish

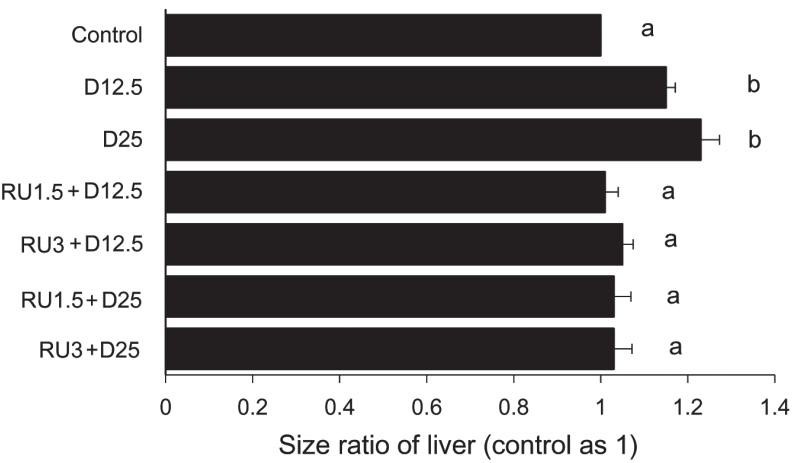

Fig. 1. Effect of RU486 on dexamethasone-induced hepatomegaly. Larval zebrafish at $5 \mathrm{dpf}$ were exposed to dexamethasone $(12.5$ and $25.0 \mu \mathrm{g} / \mathrm{mL})$ or co-exposed to dexamethasone $(12.5$ and $25.0 \mu \mathrm{g} / \mathrm{mL})$ and RU486 $(1.5$ and $3.0 \mu \mathrm{g} / \mathrm{mL})$ for $24 \mathrm{hr}$. D12.5, $12.5 \mu \mathrm{g} / \mathrm{mL}$ dexamethasone; D25, $25 \mu \mathrm{g} / \mathrm{mL}$ dexamethasone; RU1.5, $1.5 \mu \mathrm{g} / \mathrm{mL}$ RU486, RU3, $3 \mu \mathrm{g} / \mathrm{mL}$ RU486. $\mathrm{n}=10$. Values with different letters are significantly different $(\mathrm{p}<0.05)$.

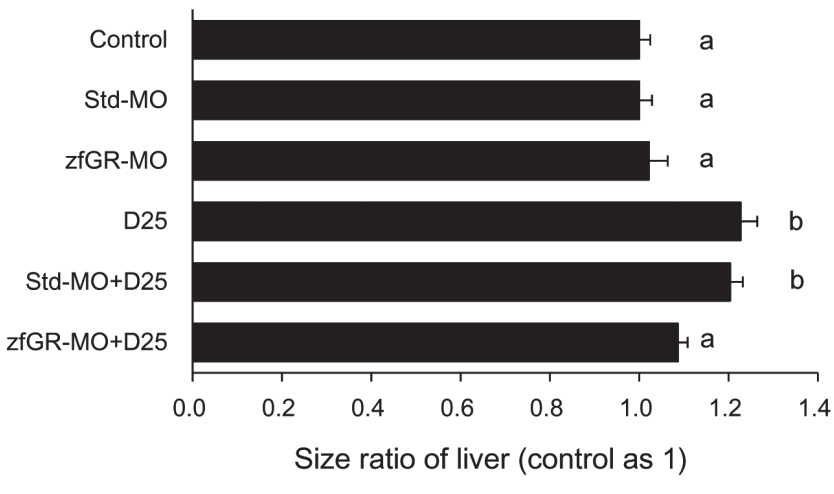

Fig. 2. Effect of GR-MO on dexamethasone-induced hepatomegaly. D25, $25.0 \mu \mathrm{g} / \mathrm{mL}$ dexamethasone; StdMO, standard morpholino; zfGR-MO, zebrafish GR morpholino. $\mathrm{n}=10$. Values with different letters are significantly different $(\mathrm{p}<0.05)$.
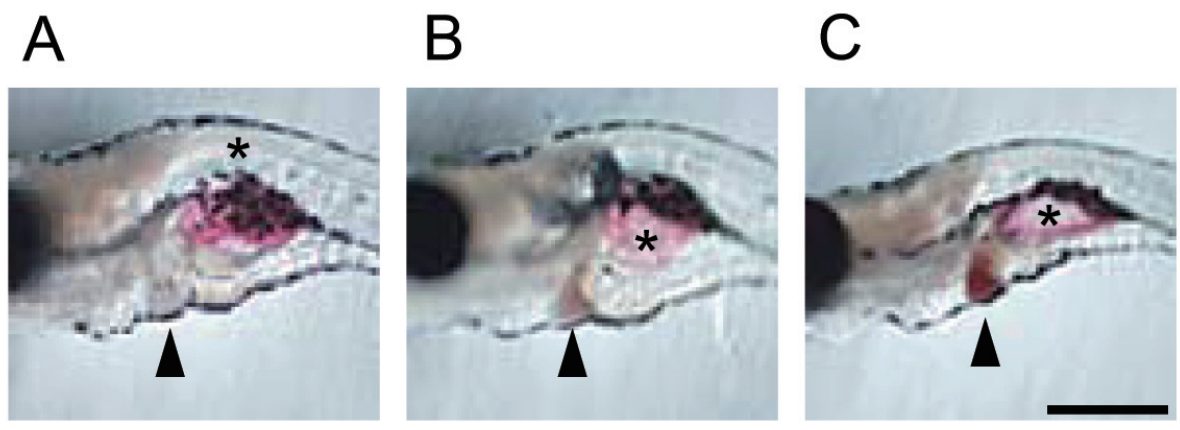

Fig. 3. Degree of steatosis in $6 \mathrm{dpf}$ zebrafish larvae. A, negative (-); B, mild (+); C, severe (++). Arrowheads indicate liver and asterisks indicate the swim bladder that was stained with oil red nonspecifically. Scale bar, $500 \mu \mathrm{m}$.

dexamethasone-induced hepatomegaly was mediated by GR.

To further confirm the involvement of zfGR in dexamethasone-induced hepatomegaly, gene knock-down with morpholino antisense oligonucleotides against zfGR was conducted. As shown in Fig. 2, dexamethasone at $25.0 \mu \mathrm{g} / \mathrm{mL}$ caused a significant increase of liver size compared to the control. Exposure of larval zebrafish developed from standard-morpholino-injected embryos to $25.0 \mu \mathrm{g} / \mathrm{mL}$ dexamethasone also caused a significant increase of liver size compared to the control. However, larval zebrafish developed from zfGR-MO-injected embryos showed no significant difference in liver size after exposure to $25.0 \mu \mathrm{g} / \mathrm{mL}$ dexamethasone. These results provide further evidence that dexamethasone-induced hepatomegaly is mediated by GR.

It has been proposed that corticosteroid-induced hepatomegaly is caused by excessive fat accumulation in the liver (Micuda et al., 2007). Oil red staining was performed to detect fat accumulation in larval zebrafish after 24-hr exposure to dexamethasone. The degree of steatosis of larval zebrafish after oil red staining was graded as negative (-), mild $(+)$ and severe $(++)$ based on the shades of red in the liver (Fig. 3). As shown in Table 1, 50\% of the larvae had negative steatosis in the control group. Treatment with $12.5 \mu \mathrm{g} / \mathrm{mL}$ dexamethasone decreased the proportion of larvae with negative steatosis from $50 \%$ to $0 \%$. Instead, the proportions of larvae with mild steato- 
sis and severe steatosis were increased to $40 \%$ and $60 \%$, respectively. Co-exposure of larvae to RU486 and dexamethasone caused dose-response decreases in the percentages of larva with mild and severe steatosis, suggesting that dexamethasone-induced hepatic steatosis was also mediated by zfGR.

In the present study, we demonstrated for the first time that dexamethasone induced hepatomegaly and fatty liver in fish and also larval fish, primarily via the zfGR. Both concentrations of RU486 inhibited hepatomegaly induced by dexamethasone $(25.0 \mu \mathrm{g} / \mathrm{mL})$ to $14.5-15.0 \%$. Knockdown by zfGR-MO also reduced $25.0 \mu \mathrm{g} / \mathrm{mL}$ dexamethasone-induced hepatomegaly to $39.1 \%$. In fish, cortisol is the major corticosteroid that is released in response to stress. The plasma concentrations of teleost cortisol rise dramatically during stress (Ellis et al., 2012). In intensive aquaculture, fish are subject to constant stress such as stress from handling, transport, and high stocking density and stress caused by toxicants, which will elevate the plasma cortisol concentration. A stressor-induced elevation of cortisol might also contribute to the development of fatty liver and hepatomegaly.

The GR is believed to be maintained as an inactive cytoplasmic complex with cofactors, such as heat shock proteins and immunophilins. Following ligand binding that subsequently dissociate the cofactors, the GR translocates to the nucleus, where it binds glucocorticoid response elements (GREs), as a dimer, to promote the transcription of responsive genes (Chivers et al., 2004). Using the responsiveness of gene expression and morphological changes to GR agonists, use of larval zebrafish as an in vivo bioassay system in monitoring environmental glucocorticoid contaminants has been suggested (Chen et al., 2016, 2017). Our studies raise the possibility that larval zebrafish could be used as a convenient model system to study the mechanisms of the development of hepatomegaly and steatosis caused by a glucocorticoid as a representative non-alcoholic fatty liver disease in humans. Thus, further studies are needed to determine whether these changes may lead to liver failure in zebrafish during development. Detailed information on the mechanism of glucocorticoid-induced liver disease is required for comparison with mammalian studies (Vegiopoulos and Herzig, 2007; Patel et al., 2011).

\section{ACKNOWLEDGMENTS}

This work was supported by JSPS KAKENHI Grant Number JP14F03777 and Special Scientific Research Funds for Central Non-profit Institutes, Chinese Academy of Fishery Sciences (2016GH05).
Conflict of interest---- The authors declare that there is no conflict of interest.

\section{REFERENCES}

Boone, A.N., Ducouret, B. and Vijayan, M.M. (2002): Glucocorticoid-induced glucose release is abolished in trout hepatocytes with elevated hsp70 content. J. Endocrinol., 172, R1-R5.

Bertrand, S., Thisse, B., Tavares, R., Sachs, L., Chaumot, A., Bardet, P.L., Escrivà, H., Duffraisse, M., Marchand, O., Safi, R., Thisse, C. and Laudet, V. (2007): Unexpected novel relational links uncovered by extensive developmental profiling of nuclear receptor expression. PLoS Genet., 3, e188.

Chen, Q., Jia, A., Snyder, S.A., Gong, Z. and Lam, S.H. (2016): Glucocorticoid activity detected by in vivo zebrafish assay and in vitro glucocorticoid receptor bioassay at environmental relevant concentrations. Chemosphere, 144, 1162-1169.

Chen, Q., Li, C., Gong, Z., Chan, E.C., Snyder, S.A. and Lam, S.H (2017): Common deregulated gene expression profiles and morphological changes in developing zebrafish larvae exposed to environmental-relevant high to low concentrations of glucocorticoids. Chemosphere, 172, 429-439.

Chivers, J.E., Cambridge, L.M., Catley, M.C., Mak, J.C., Donnelly, L.E., Barnes, P.J. and Newton, R. (2004): Differential effects of RU486 reveal distinct mechanisms for glucocorticoid repression of prostaglandin $\mathrm{E}_{2}$ release. Eur. J. Biochem., 271, 4042-4052.

Ellis, T., Yildiz, H.Y., López-Olmeda, J., Spedicato, M.T., Tort, L., Øverli, Ø. and Martins, C.I. (2012): Cortisol and finfish welfare. Fish Physiol. Biochem., 38, 163-188.

Howarth, D.L., Yin, C., Yeh, K. and Sadler, K.C. (2013): Defining hepatic dysfunction parameters in two models of fatty liver disease in zebrafish larvae. Zebrafish, 10, 199-210.

Liu, Q., Tan, Q.S., Chen, X.X., Du, Y.D., Xia, J., Yang, Q. and Ma, Y.X. (2009): Changes of biochemical characteristics and organization structure in liver of grass carp (Ctenopharyngodon idellus) with hepatobiliary syndrome. J. Anhui Agric. Sci., 14, 64636467.

Mathew, L.K., Sengupta, S., Kawakami, A., Andreasen, E.A., Löhr, C.V., Loynes, C.A., Renshaw, S.A., Peterson, R.T. and Tanguay, R.L. (2007): Unraveling tissue regeneration pathways using chemical genetics. J Biol. Chem., 282, 35202-35210.

Micuda, S., Fuksa, L., Mundlova, L., Osterreicher, J., Mokry, J., Cermanova, J., Brcakova, E., Staud, F., Pokorna, P. and Martinkova, J. (2007): Morphological and functional changes in P-glycoprotein during dexamethasone-induced hepatomegaly. Clin. Exp. Pharmacol. Physiol., 34, 296-303.

Nijoukubo, D., Tanaka, Y., Okuno, Y., Yin, G., Kitazawa, T., Peterson, R.E., Kubota, A. and Teraoka, H. (2016) :Protective effect of prostacyclin against pre-cardiac edema caused by 2,3,7,8-tetrachlorodibenzo- $p$-dioxin and a thromboxane receptor agonist in developing zebrafish. Chemosphere, 156, 111-117.

Patel, R., Patel, M., Tsai, R., Lin, V., Bookout, A.L., Zhang, Y., Magomedova, L., Li, T., Chan, J.F. and Budd, C. (2011): LXR $\beta$ is required for glucocorticoid-induced hyperglycemia and hepatosteatosis in mice. J. Clin. Invest., 121, 431-441.

Raldúa, D., André, M. and Babin, P.J. (2008): Clofibrate and gemfibrozil induce an embryonic malabsorption syndrome in zebrafish. Toxicol. Appl. Pharmacol., 228, 301-314.

Salas-Leiton, E., Coste, O., Asensio, E., Infante, C., Cañavate, J.P. and Manchado, M. (2012): Dexamethasone modulates expres- 
Hepatomegaly in larval zebrafish

sion of genes involved in the innate immune system, growth and stress and increases susceptibility to bacterial disease in Senegalese sole (Solea senegalensis Kaup, 1858). Fish Shellfish Immunol., 32, 769-778.

Thatcher, N.J. and Caldwell, J. (1994): Origins of hepatomegaly pro- duced by dexamethasone (DEX), pregnenolone $16 \alpha$-carbonitrile $(\mathrm{PCN})$ and phenobarbitone $(\mathrm{PB})$ in female Sprague-Dawley rats. Biochem. Soc. Trans., 22, 132S.

Vegiopoulos, A. and Herzig, S. (2007): Glucocorticoids, metabolism and metabolic diseases. Mol. Cell. Endocrinol., 275, 43-61. 